\title{
ORIGINAL
}

\section{Tracheostomy practice and timing in traumatic brain-injured patients: a CENTER-TBI study}

\author{
Chiara Robba 1,2 ${ }^{10}$, Stefania Galimberti ${ }^{2,3}$, Francesca Graziano ${ }^{2,3}$, Eveline J. A. Wiegers ${ }^{4}$, Hester F. Lingsma ${ }^{4}$, \\ Carolina laquaniello, Nino Stocchetti ${ }^{5,6}$, David Menon ${ }^{7}$, Giuseppe Citerio ${ }^{2,8^{*}}$ (ID and The CENTER-TBI ICU \\ Participants and Investigators
}

(c) 2020 Springer-Verlag GmbH Germany, part of Springer Nature

\begin{abstract}
Purpose: Indications and optimal timing for tracheostomy in traumatic brain-injured (TBI) patients are uncertain. This study aims to describe the patients' characteristics, timing, and factors related to the decision to perform a tracheostomy and differences in strategies among different countries and assess the effect of the timing of tracheostomy on patients' outcomes.

Methods: We selected TBI patients from CENTER-TBI, a prospective observational longitudinal cohort study, with an intensive care unit stay $\geq 72 \mathrm{~h}$. Tracheostomy was defined as early ( $\leq 7$ days from admission) or late ( $>7$ days). We used a Cox regression model to identify critical factors that affected the timing of tracheostomy. The outcome was assessed at 6 months using the extended Glasgow Outcome Score.

Results: Of the 1358 included patients, 433 (31.8\%) had a tracheostomy. Age (hazard rate, $\mathrm{HR}=1.04,95 \% \mathrm{Cl}=1.01-$ $1.07, p=0.003)$, Glasgow coma scale $\leq 8(H R=1.70,95 \% \mathrm{Cl}=1.22-2.36$ at $7 ; p<0.001)$, thoracic trauma $(\mathrm{HR}=1.24$, $95 \% \mathrm{Cl}=1.01-1.52, p=0.020)$, hypoxemia $(H R=1.37,95 \% \mathrm{Cl}=1.05-1.79, p=0.048)$, unreactive pupil $(\mathrm{HR}=1.76$, $95 \% \mathrm{Cl}=1.27-2.45$ at $7 ; p<0.001)$ were predictors for tracheostomy. Considerable heterogeneity among countries was found in tracheostomy frequency (7.9-50.2\%) and timing (early 0-17.6\%). Patients with a late tracheostomy were more likely to have a worse neurological outcome, i.e., mortality and poor neurological sequels $(O R=1.69,95 \%$ $\mathrm{Cl}=1.07-2.67, p=0.018$ ), and longer length of stay (LOS) (38.5 vs. 49.4 days, $p=0.003$ ).

Conclusions: Tracheostomy after TBI is routinely performed in severe neurological damaged patients. Early tracheostomy is associated with a better neurological outcome and reduced LOS, but the causality of this relationship remains unproven.
\end{abstract}

Keywords: Traumatic Brain Injury, Tracheostomy, Mechanical ventilation, Outcome

\footnotetext{
*Correspondence: giuseppe.citerio@unimib.it

${ }^{2}$ School of Medicine and Surgery, University of Milano - Bicocca, Monza, Italy

Full author information is available at the end of the article

Chiara Robba and Stefania Galimberti have contributed equally to this work.

CENTER-TBI ICU Participants and Investigators are listed as contributors at the end of the manuscript.
}

\section{国 Springer}




\section{Introduction}

Tracheostomy can facilitate weaning in long-term ventilated patients, potentially shortening the duration of mechanical ventilation and intensive care unit (ICU) stay, and reducing complications from prolonged tracheal intubation, such as ventilator-associated pneumonia (VAP) and tracheal lesions [1]. In patients who require ICU care after a TBI, the main indications for tracheostomy include failure to wean invasive mechanical ventilation, absence of protective airway reflexes, impairment of respiratory drive, and difficulties in managing secretions [2]. The proportion of TBI patients who might benefit from a tracheostomy, and the most appropriate timing for the procedure [3] are still undefined, and relevant biases confound the limited, mainly retrospective, available data on this issue. Moreover, policies and clinical practice vary among different centres, and the optimal indications for tracheostomy remain uncertain [4].

Conventionally, tracheostomies performed in the first week are classified as early, while tracheostomies performed later than 7 days are defined as late [5]. The ideal timing for a tracheostomy is uncertain since the evidence on the advantages of early over late tracheostomy is conflicting, and no real differences in mortality have been identified between early and late tracheostomy so far $[6$, 7].

To obtain insights into tracheostomy in patients who had suffered a TBI, we analysed data from the ICU stratum of the CENTER-TBI study [8]. This study aims to describe the characteristics of those TBI patients who undergo a tracheostomy and the current state of its timing; to identify the factors involved in performing the procedure and the different strategies between countries, and to assess the effect of the timing on patients' outcome.

\section{Methods}

The Collaborative European NeuroTrauma Effectiveness Research in Traumatic Brain Injury (CENTER-TBI study, registered at clinicaltrials.gov NCT02210221) is a longitudinal prospective collection of TBI patient data across 65 centres in Europe between December 19, 2014, and December 17, 2017, as previously described $[8,9]$. The Medical Ethics Committees approved the CENTERTBI study in all participating centres, and we obtained informed consent according to local regulations.

We performed a pre-planned analysis focusing on tracheostomy practice in the CENTER-TBI cohort during the ICU stay (ESM1). The project was preregistered on the CENTER-TBI proposal platform in December 2018 and approved by the CENTER-TBI proposal review committee (ESM Document 1) before starting the analysis.

\section{Take-home message}

Tracheostomy after TBI is commonly performed in the most severe neurological damaged patients. Early tracheostomy is associated with shorter ICU length of stay and with a trend of a better outcome.

This report complies with the Strengthening the Reporting of Observational Studies in Epidemiology (STROBE) reporting guidelines (ESM Table S1).

For this analysis, the inclusion criteria were:

- A clinical diagnosis of TBI with an indication for a brain Computed Tomography scan (CT);

- Presentation to the hospital within $24 \mathrm{~h}$ (hrs) postinjury;

- ICU admission with a length of stay $(\mathrm{LOS}) \geq 72 \mathrm{~h}$.

Exclusion criteria were:

- Death in the first $72 \mathrm{~h}$;

- Short ICU LOS (<72 h).

These exclusion criteria were defined to exclude patients in whom tracheostomy was never likely to have been considered, either because of extremely severe injury and rapid death, or those in whom the injury was not severe enough.

\section{Data collection}

Detailed data were collected on pre-injury factors and patient's characteristics, injury details, Glasgow coma scale (GCS), pre-hospital care, clinical care, post-acute care, and outcome, with a total of over 2500 unique data fields, with many fields collected serially over time (e.g., physiological variables in the ICU stratum). Hypoxemia was defined as a documented partial pressure of oxygen $\left(\mathrm{PaO}_{2}\right)<8 \mathrm{kPa}(60 \mathrm{mmHg})$, oxygen saturation $\left(\mathrm{SaO}_{2}\right)<90 \%$, or both; hypotension was defined as a documented systolic blood pressure $<90 \mathrm{mmHg}$.

\section{Objectives}

The aim of this study is threefold:

1. Describe the patients' characteristics and timing of tracheostomy in TBI patients;

2. Identify the factors related to the decision to perform a tracheostomy and differences in strategies among different countries;

3. Assess the effect of the timing of tracheostomy on patients' outcomes. 


\section{Outcomes}

The primary endpoint was the patients' functional outcome assessed by the Extended Glasgow Outcome Score (GOSE) at 6 months. An unfavourable outcome was defined as GOSE $\leq 4$, which takes into account both poor neurological outcome and mortality together. All responses were obtained by study personnel from patients or from a proxy (where impaired cognitive capacity prevented patient interview), during a face-to-face visit, by telephone interview, or by postal questionnaire at 6 months (range 5-8 months) after injury [10]. All outcome evaluators had received training in the use of the GOSE. We also registered mortality at 6 months, and the ICU and hospital LOS.

\section{Statistical analysis}

Continuous variables are described with median and interquartile range (IQR), or mean and standard deviation (SD), as appropriate, and categorical data were reported as absolute and relative frequencies. The nature of the variables guided the choice of the test for the comparison among groups.

\section{Factors related to the decision to perform a tracheostomy}

A Cox regression model was used to identify the key factors that affected the decision and timing of tracheostomy during ICU stay. Time origin was ICU admission, and patients who did not receive the procedure were censored at discharge from ICU or at death, whichever occurred first. A frailty term was included to account for centre-specific effects. Variables significant in the univariate analysis, and others judged clinically relevant, were initially identified, and the selection of the covariates for the final model (including age, GCS, pupillary reactivity, hypoxemia, thoracic, and facial trauma) was based on the likelihood ratio test (LRT) and Akaike Information Criterion (AIC). Assumptions regarding the proportionality of the hazards and the linearity of effects were investigated using the Schoenfeld test and the Martingale residuals, respectively [11]. For variables violating the proportional hazards assumption, the time dependence of the effect was adjusted by including a term for the interaction of the variable and time [11].

\section{Country and centre differences}

The country- and centre-specific incidence rate of late, early, and no tracheostomy was estimated from a proportional odds model, adjusting for patient characteristics associated with a tracheostomy, and including a random intercept for country and centre. The median odds ratio
(MOR) was also calculated as a measure of variability between centres [12].

\section{Outcomes}

The role of timing of tracheostomy on different outcomes was explored on the subset of patients who underwent a tracheostomy. The time to the procedure was evaluated both as a discrete (i.e., days from ICU admission) and as a categorical variable (i.e., $\leq 7$ vs. $>7$ days) [4]. A logistic regression model was applied to the odds of an unfavourable GOSE (GOSE $\leq 4)$, while we performed a Cox model on the 6-month mortality from ICU admission, with patients contributing to the risk set from the day of tracheostomy. Death from any cause was the event of interest, and patients alive at 6 months from ICU admission were censored. A linear regression model was used for the evaluation of LOS in both ICU and hospital. LOS was calculated from ICU admission (and from tracheostomy) to discharge or death in ICU, with a sensitivity analysis that excluded patients who died in ICU or hospital. All analyses were adjusted for known outcome predictors in the Core IMPACT model (i.e., age, GCS at arrival, and pupillary reactivity) [13].

\section{Missing values}

We used a multivariate imputation by chained equations in all the multivariable models to deal with missing values in the predictors, generating 50 imputed datasets [14]. Analyses on complete cases were also performed to check consistency in the results. Model diagnostics were performed in all the imputed datasets, and final decisions were taken based on the findings of the majority of datasets.

All the tests conducted were two-sided with a significance level of $5 \%$. The analyses were conducted in R (version 3.5.2, R Core Team, 2019) [15].

\section{Results}

Of the 2138 consecutive patients requiring ICU care, 1358 (from 19 countries and 54 centres) had an ICU LOS $\geq 72 \mathrm{~h}$. Of these, 433 subjects $(31.8 \%$ of the study cohort, $20.2 \%$ of the overall ICU population) underwent a tracheostomy and were included in the analysis (ESM Figure S1). Details regarding the screening and enrolment process are described in the main CENTER-TBI manuscript [9].

\section{Patients' characteristics}

Patients' characteristics at ICU admission are summarized in Table 1 (both overall and stratified by whether or not they received a tracheostomy). Patients who received or did not receive a tracheostomy were similar in terms of age, sex, pre-injury American Society 
Table 1 Features at admission and during ICU stay in patients who received and did not receive tracheostomy and the overall population

\begin{tabular}{|c|c|c|c|c|c|}
\hline Characteristic & $\begin{array}{l}\text { No tracheostomy } \\
(n=925)\end{array}$ & $\begin{array}{l}\text { Tracheostomy } \\
(n=433)\end{array}$ & $P$ value & Overall ( $n=1358)$ & $n$ missing \\
\hline \multicolumn{6}{|l|}{ At admission } \\
\hline Age (years), median (I-III quartiles) & $50(29-65)$ & $45(29-63)$ & 0.102 & $49(29-64)$ & 0 \\
\hline Age $\geq 65$ years, $n(\%)$ & $232(25.1)$ & $99(22.9)$ & 0.413 & $331(24.4)$ & \\
\hline Sex: male, $n(\%)$ & $677(73.2)$ & $333(76.9)$ & 0.163 & $1010(74.4)$ & 0 \\
\hline Race: caucasian, $n(\%)$ & $799(97.3)$ & $377(95.9)$ & 0.260 & $1176(96.9)$ & 144 \\
\hline Pre-injury ASAPS, $n(\%)$ & & & 0.235 & & 75 \\
\hline Normal healthy patient & $489(56.7)$ & $257(61.2)$ & & $746(58.1)$ & \\
\hline Patient with mild systemic disease & $278(32.2)$ & $126(30)$ & & $404(31.5)$ & \\
\hline Patient with severe systemic disease & $96(11.1)$ & $37(8.8)$ & & $133(10.4)$ & \\
\hline Cause of injury, $n(\%)$ & & & 0.229 & & 58 \\
\hline Road traffic accident & $401(45.7)$ & $215(50.8)$ & & $616(47.4)$ & \\
\hline Incidental fall & $360(41)$ & $148(35)$ & & $508(39.1)$ & \\
\hline Violence/assault & $33(3.8)$ & $18(4.3)$ & & $51(3.9)$ & \\
\hline Suicide attempt & $15(1.7)$ & $11(2.6)$ & & $26(2)$ & \\
\hline Other & $68(7.8)$ & $31(7.3)$ & & $99(7.6)$ & \\
\hline ISS, mean (SD) & $33.45(14)$ & $38.40(14.6)$ & $<0.001$ & $35.05(14.4)$ & 21 \\
\hline$I S S \geq 25, n(\%)$ & $552(61)$ & $305(70.6)$ & 0.001 & $857(64.1)$ & \\
\hline Alcohol involved, $n(\%)$ & $245(30.2)$ & $102(27.6)$ & 0.392 & $347(29.4)$ & 177 \\
\hline Drug abuse, $n(\%)$ & $28(3.9)$ & $31(9.4)$ & 0.001 & $59(5.6)$ & 303 \\
\hline Hypoxemia: yes or suspected, $n$ (\%) & $111(13)$ & $78(19.5)$ & 0.004 & $189(15.1)$ & 105 \\
\hline Hypotension: yes or suspected, $n(\%)$ & $102(12)$ & $86(21.1)$ & $<0.001$ & $188(14.9)$ & 97 \\
\hline Severity TBI, $n(\%)$ & & & $<0.001$ & & 85 \\
\hline Mild & $264(30.6)$ & $53(12.9)$ & & $317(24.9)$ & \\
\hline Moderate & $144(16.7)$ & $64(15.6)$ & & $208(16.3)$ & \\
\hline Severe & $454(52.7)$ & $294(71.5)$ & & $748(58.8)$ & \\
\hline Pupillary reactivity, $n$ (\%) & & & $<0.001$ & & 82 \\
\hline Both reactive & $732(84.8)$ & $299(72.4)$ & & $1031(80.8)$ & \\
\hline One reactive & $52(6)$ & $42(10.2)$ & & $94(7.4)$ & \\
\hline Both unreactive & $79(9.2)$ & $72(17.4)$ & & $151(11.8)$ & \\
\hline GCS, median (I-III quartile) & $8(3-13)$ & $5(3-9)$ & $<0.001$ & $7(3-12)$ & 85 \\
\hline Any extra-cranial injury, n (\%) & $525(56.8)$ & $291(67.2)$ & $<0.001$ & $816(60.1)$ & 0 \\
\hline Facial trauma, $n(\%)$ & $210(22.7)$ & $128(29.6)$ & 0.008 & $338(24.9)$ & 0 \\
\hline Thoracic trauma, $n(\%)$ & $339(36.6)$ & $206(47.6)$ & $<0.001$ & $545(40.1)$ & 0 \\
\hline \multicolumn{6}{|l|}{$\ln I C U$} \\
\hline Cranial surgery, $n(\%)$ & $364(39.8)$ & $261(60.4)$ & $<0.001$ & $625(46.4)$ & 11 \\
\hline Extra-cranial surgery, $n(\%)$ & $236(25.8)$ & $227(52.5)$ & $<0.001$ & $463(34.3)$ & 10 \\
\hline Reintubation, $n(\%)$ & $65(7.3)$ & $50(11.7)$ & 0.010 & $115(8.7)$ & 40 \\
\hline Ventilator acquired pneumonia, $n(\%)$ & $127(14)$ & $149(34.5)$ & $<0.001$ & $276(20.6)$ & 16 \\
\hline ICP monitor, $n(\%)$ & $478(52.4)$ & $351(81.1)$ & $<0.001$ & $829(61.6)$ & 12 \\
\hline Respiratory failure, $n(\%)$ & $220(24.2)$ & $207(47.8)$ & $<0.001$ & $427(31.8)$ & 15 \\
\hline Antibiotics used, $n(\%)$ & $724(83.7)$ & $401(94.8)$ & $<0.001$ & $1490(74.4)$ & 0 \\
\hline
\end{tabular}

ASAPS American Society of Anaesthesiologists' Physical Status, ICP intracranial pressure, ISS injury severity score, TBI Traumatic Brain Injury

of Anaesthesiologists' physical status (ASAPS) score, mechanism of injury, and pre-injury clinical history. Patients receiving tracheostomy more frequently had lower median GCS at arrival (median 5 vs. $8, p<0.001$ ), and abnormal pupillary reactivity (at least one unreactive pupil in $27.6 \%$ vs. $15.2 \%, p<0.001$ ). Moreover, patients who underwent tracheostomy had a higher rate of early hypoxemia ( $19.5 \%$ vs. $13.0 \%, p=0.004)$, early hypotension 
(21.1\% vs. $12.0 \%, p<0.001)$ and higher Injury Severity Score (ISS; mean of 38.4 vs. 33.5, $p<0.001$ ) due to more extra-cranial traumatic injury $(67.2 \%$ vs. $56.8 \%, p<0.001)$, especially facial ( $29.6 \%$ vs. $22.7 \%, p=0.008)$ and thoracic trauma $(47.6 \%$ vs. $36.6 \%, p<0.001)$.

During their ICU stay, patients receiving tracheostomy more frequently underwent the placement of an intracranial pressure (ICP) monitoring device $(81.1 \%$ vs. 52.4 , $p<0.001)$, and suffered from ventilator acquired pneumonia (VAP; $35.5 \%$ vs. $14.0 \%, p<0.001$ ), and respiratory failure $(47.8 \%$ vs. $24.2 \%, p<0.001)$ (Table 1$)$. Of the 1358 patients included in the study, $96(7 \%)$ received a withdrawal of treatment: 86 (9.3\%) were not tracheotomised, and $10(2.3 \%)$ had undergone a tracheostomy.

\section{Timing of tracheostomy}

The median (IQR) time to tracheostomy of the 433 patients was 9 (5-14) days from ICU admission, with 30 (6.9\%) of the patients receiving tracheostomy on the day of ICU admission and the last procedure performed after 39 days in ICU (ESM Figure S2 and Figure S3). Details on the characteristics of the tracheotomised patients are reported separately for early (180 patients, $41.6 \%)$ and late (253 patients, $58.4 \%$ ) procedures in Table 2. Patients receiving early tracheostomies were older $(30.6 \%$ vs. $17.4 \%$ aged $\geq 65$ years, $p=0.002$ ), with a higher incidence of hypoxemia $(24.4 \%$ vs. $16.1 \%, p=0.054)$ and hypotension $(25.9 \%$ vs. $17.6 \%, p=0.059)$ in the pre-hospital and emergency department settings, and had facial injuries (34.4\% vs. $26.1 \%, p=0.076)$. Patients receiving a late tracheostomy had a higher rate of ventilator-associated pneumonia (39.7\% vs. $27.2 \%, p=0.01)$, and respiratory failure (52.2\% vs. $41.7 \%, p=0.039)$.

\section{Factors related to the decision to perform a tracheostomy}

The results of the Cox regression model for the tracheostomy procedure are reported in Table 3 . Age had a statistically significant impact, indicating a $4 \%$ increase in the hazard of tracheostomy for each 5 year increase in age $(\mathrm{HR}=1.04,95 \% \mathrm{CI}=1.01-1.07, p=0.003)$. The hazard for requiring a tracheostomy was significantly lower in patients with GCS $>8$ vs. those with $\mathrm{GCS} \leq 8(p<0.001)$ and the HR increased linearly after ICU admission, with the $\mathrm{HR}$ at 1,7 and 15 days from admission calculated as 1.51 (95\% CI $=1.09-2.10), 1.70$ (95\% CI $=1.22-2.36)$, and $1.98(95 \% \mathrm{CI}=1.42-2.75)$, respectively. The effect of pupillary reactivity was also not constant in time, and the HR estimates indicate that patients with at least one unreactive pupil have a higher hazard $(p<0.001)$ as compared to those with both reacting pupils, with an HR at 1,7 and 15 days from admission of $1.63(95 \% \mathrm{CI}=1.17$ 2.27), $1.76(95 \% \mathrm{CI}=1.27-2.45)$ and $1.96(95 \% \mathrm{CI}=1.41-$ 2.72). The hazard of tracheostomy was 1.24 times higher in patients with thoracic trauma as compared to those without (95\% CI $=1.01-1.52, p=0.020$ ), while the two timing groups did not show a significant difference in the incidence of facial trauma $(\mathrm{HR}=1.24,95 \% \mathrm{CI}=1.00$ 1.53, $p_{\mathrm{LRT}}=0.0714$, and $\left.p=0.149\right)$. Finally, hypoxemia was associated with an increased hazard of undergoing a tracheostomy ( $\mathrm{HR}=1.37,95 \% \mathrm{CI}=1.05-1.79, p=0.048)$. The findings of the model on complete cases were consistent (ESM Table S2).

\section{Country and centre differences}

We observed a considerable heterogeneity among countries in the decision to perform a tracheostomy (with adjusted tracheostomy rates ranging from 7.9 to $50.2 \%$ ) and in the timing for tracheostomy (with the incidence of late tracheostomy ranging from 7.9 to $32.6 \%$, and early tracheostomy from 0 to 17.6\%) (Fig. 1a). Furthermore, individual centres within the same country showed different adjusted percentages of early vs. late tracheostomy (Fig. 1b). In the vast majority of centres, a delayed procedure was more likely to happen than an early one, and only in two institutions, the policy was to opt exclusively for an early strategy. Moreover, the variability in the centre-specific rate of late tracheostomy was more pronounced than the early rate. The crude rates observed at country and centre levels are shown in ESM Figure S4. We used the MOR to quantify between-centre differences and found that even after correction for patient characteristics, there was a 2.2-fold difference in the odds of tracheostomy between centres with the highest and lowest tracheostomy rates.

\section{Outcomes}

The univariate analyses (ESM Table S3) showed no significant effect of early vs. late tracheostomy on ICU mortality, 6-month mortality, or 6-month GOSE $(p=0.399$, $p=0.735$, and $p=0.197$, respectively). However, patients who received a late tracheostomy had a statistically significant longer mean LOS in ICU (19.6 vs. 26.7 days, $p<0.001)$ and in hospital (38.5 vs. 49.4 days, $p=0.003$ ) when measured from the point of ICU admission. These differences were abolished when LOS was measured from tracheostomy (mean LOS in ICU for early vs. late tracheostomy: 14.8 days vs. 12.5 days, $p=0.045$; mean LOS in hospital: 13.1 days vs. 34.7 days, $p=0.915$ ).

The adjusted regression analyses demonstrated an association between an early tracheostomy and a better neurological outcome captured by the GOSE (Table 4). Patients with a late tracheostomy were more likely to have a worse neurological outcome (Model 1: $\mathrm{OR}=1.69,95 \% \mathrm{CI}=1.07-2.67, p=0.018)$, and the analysis using day to tracheostomy as a continuous variable (Model 2) showed that every day of delay in performing 
Table 2 Features at admission and during ICU stay for early and late tracheostomy

\begin{tabular}{|c|c|c|c|c|}
\hline Characteristic & $\begin{array}{l}\text { Early tracheostomy } \\
(n=180)\end{array}$ & $\begin{array}{l}\text { Late tracheostomy } \\
(n=253)\end{array}$ & $P$ value & $n$ missing \\
\hline Age (years), median (I-III quartiles) & $48.5(31-67)$ & $44.0(28-59)$ & 0.024 & 0 \\
\hline Age $\geq 65$ years, $n(\%)$ & $55(30.6)$ & $44(17.4)$ & 0.002 & \\
\hline Sex: male, $n(\%)$ & $139(77.2)$ & $194(76.7)$ & 0.987 & 0 \\
\hline Pre-injury ASAPS, $n(\%)$ & & & 0.948 & 13 \\
\hline Normal healthy patient & $105(60.3)$ & $152(61.8)$ & & \\
\hline Patient with mild systemic disease & $53(30.5)$ & $73(29.7)$ & & \\
\hline Patient with severe systemic disease & $16(9.2)$ & $21(8.5)$ & & \\
\hline Previous TBI, $n(\%)$ & $12(7.5)$ & $15(6.5)$ & 0.833 & 42 \\
\hline Use of anticoagulants, $n$ (\%) & $9(5.2)$ & $8(3.3)$ & 0.465 & 18 \\
\hline Use of antiplatelets' drugs, $n$ (\%) & $18(10.5)$ & $19(7.8)$ & 0.449 & 18 \\
\hline Hypoxemia:Yes or Suspected, $n$ (\%) & $40(24.4)$ & $38(16.1)$ & 0.054 & 33 \\
\hline Hypotension: Yes or Suspected, $n$ (\%) & $44(25.9)$ & $42(17.6)$ & 0.059 & 25 \\
\hline Cardiovascular history, $n$ (\%) & $45(25.6)$ & $52(21.1)$ & 0.343 & 11 \\
\hline ISS, mean (SD) & $38.3(14.8)$ & $38.5(14.5)$ & 0.896 & 1 \\
\hline$I S S \geq 25, n(\%)$ & $128(71.1)$ & $177(70.2)$ & 0.929 & \\
\hline Severity of TBI, $n(\%)$ & & & 0.863 & 22 \\
\hline Mild & $22(13.1)$ & $31(12.8)$ & & \\
\hline Moderate & $28(16.7)$ & $36(14.8)$ & & \\
\hline Severe & $118(70.2)$ & $176(72.4)$ & & \\
\hline Cause of injury, $n(\%)$ & & & 0.511 & 10 \\
\hline Road traffic accident & $90(51.4)$ & $125(50.4)$ & & \\
\hline Incidental fall & $56(32)$ & $92(37.1)$ & & \\
\hline Suicide attempt & $7(4)$ & $4(1.6)$ & & \\
\hline Violence/assault & $8(4.6)$ & $10(4.0)$ & & \\
\hline Other & $14(8)$ & $17(6.9)$ & & \\
\hline Alcohol involved, $n(\%)$ & $39(25.7)$ & $63(28.9)$ & 0.570 & 63 \\
\hline Drugs involved, $n(\%)$ & $13(9.5)$ & $18(9.3)$ & 1.000 & 102 \\
\hline Pupillary reactivity, 4 (\%) & & & 0.675 & 20 \\
\hline Both reactive & $120(70.6)$ & $179(73.7)$ & & \\
\hline One reactive & $17(10)$ & $25(10.3)$ & & \\
\hline Both unreactive & $33(19.4)$ & $39(16)$ & & \\
\hline GCS, median (I-III quartile) & $5.50(3-10)$ & $5(3-9)$ & 0.934 & 22 \\
\hline Any extra-cranial injury, $n$ (\%) & $121(67.2)$ & $170(67.2)$ & 1.000 & 0 \\
\hline Facial trauma, $n(\%)$ & $62(34.4)$ & $66(26.1)$ & 0.076 & 0 \\
\hline Thoracic trauma, $n(\%)$ & $84(46.7)$ & $122(48.2)$ & 0.825 & 0 \\
\hline Cranial surgery, $n(\%)$ & $102(56.7)$ & $159(63.1)$ & 0.212 & 1 \\
\hline Extra-cranial surgery, $n(\%)$ & $96(53.3)$ & $131(52)$ & 0.858 & 1 \\
\hline Reintubation, $n(\%)$ & $13(7.4)$ & $37(14.8)$ & 0.029 & 1 \\
\hline Days with tracheostomy, median (I-III quartiles) & $12.0(6.8-18.3)$ & $12.0(6-20)$ & 0.795 & 0 \\
\hline Tracheostomy at discharge from hospital, $n$ (\%) & $96(53.3)$ & $131(51.8)$ & 0.825 & 0 \\
\hline Intubated, $n(\%)$ & $173(96.6)$ & $246(97.2)$ & 0.948 & 1 \\
\hline Ventilator-associated pneumonia, $n$ (\%) & $49(27.2)$ & $100(39.7)$ & 0.010 & 1 \\
\hline ICP monitoring, $n(\%)$ & $138(76.7)$ & $213(84.2)$ & 0.065 & 0 \\
\hline Cardiac arrest, $n(\%)$ & $25(13.9)$ & $29(11.5)$ & 0.545 & 0 \\
\hline Respiratory failure, $n(\%)$ & $75(41.7)$ & $132(52.2)$ & 0.039 & 0 \\
\hline Marshall score, $n(\%)$ & & & 0.757 & 77 \\
\hline 1 & $7(4.9)$ & $10(4.7)$ & & \\
\hline 2 & $67(46.9)$ & $87(40.8)$ & & \\
\hline 3 & $16(11.2)$ & $28(13.1)$ & & \\
\hline
\end{tabular}


Table 2 (continued)

\begin{tabular}{|c|c|c|c|c|}
\hline Characteristic & $\begin{array}{l}\text { Early tracheostomy } \\
(n=180)\end{array}$ & $\begin{array}{l}\text { Late tracheostomy } \\
(n=253)\end{array}$ & $P$ value & $n$ missing \\
\hline 4 & $1(0.7)$ & $5(2.3)$ & & \\
\hline 5 & $1(0.7)$ & $1(0.5)$ & & \\
\hline 6 & $51(35.7)$ & $82(38.5)$ & & \\
\hline Antibiotics used, $n(\%)$ & $159(90.3)$ & $242(98)$ & 0.001 & 10 \\
\hline $\mathrm{H}_{2}$ Receptor antagonist used, $n$ (\%) & $40(22.7)$ & $87(35.2)$ & 0.008 & 10 \\
\hline Neuromuscular blockade used, $n$ (\%) & $78(44.3)$ & $140(56.7)$ & 0.016 & 10 \\
\hline PPI used, $n(\%)$ & $108(61.4)$ & $147(59.5)$ & 0.778 & 10 \\
\hline Prokinetics used, $n$ (\%) & $89(50.6)$ & $148(59.9)$ & 0.070 & 10 \\
\hline Sedation used, $n(\%)$ & $170(96.6)$ & $243(98.4)$ & 0.385 & 10 \\
\hline Steroids used, $n(\%)$ & $42(23.9)$ & $83(33.6)$ & 0.040 & 10 \\
\hline
\end{tabular}

ASAPS American Society of Anaesthesiologists' Physical Status, GCS glasgow coma scale, ICP intracranial pressure, ISS injury severity score, PPI proton-pump inhibitor, TBI Traumatic Brain Injury

\section{Table 3 Results of the Cox regression model for tracheostomy}

\begin{tabular}{|c|c|c|c|c|c|}
\hline \multirow[t]{2}{*}{ Variables } & \multicolumn{5}{|l|}{$\mathrm{HR}(95 \% \mathrm{Cl})$} \\
\hline & All days & $P$ value & At day 1 & At day 7 & At day 15 \\
\hline Age (5 years) & $1.04(1.01-1.07)$ & 0.003 & & & \\
\hline Thoracic trauma: yes vs. no & $1.24(1.01-1.52)$ & 0.020 & & & \\
\hline Facial trauma: yes vs. no & $1.24(1.00-1.53$ & 0.149 & & & \\
\hline Hypoxemia: yes vs. no & $1.37(1.05-1.79)$ & 0.048 & & & \\
\hline $\mathrm{GCS} \geq 8$ vs. GCS $<8^{\mathrm{a}}$ & & $<0.001 \wedge$ & $1.51(1.09-2.10)$ & $1.70(1.22-2.36)$ & $1.98(1.42-2.75)$ \\
\hline $\begin{array}{l}\text { Pupillary: } 1-2 \text { unreactive vs. } 2 \\
\text { reacting }\end{array}$ & & $<0.001 \wedge$ & $1.63(1.17-2.27)$ & $1.76(1.27-2.45)$ & $1.96(1.41-2.72)$ \\
\hline
\end{tabular}

\section{Significant random effect for center $(p<0.001)$}

GCS glasgow coma scale, SE standard error

$\wedge p$ value of the test for the overall effect: main effect + interaction of the main effect with time

a The coefficients (SE) of the model for the main effect is $0.395(0.178)$ and for the interaction with time is $0.019(0.013)$

b The coefficients (SE) of the model for the main effect is 0.474 (0.178) and for the interaction with time is $0.013(0.013)$
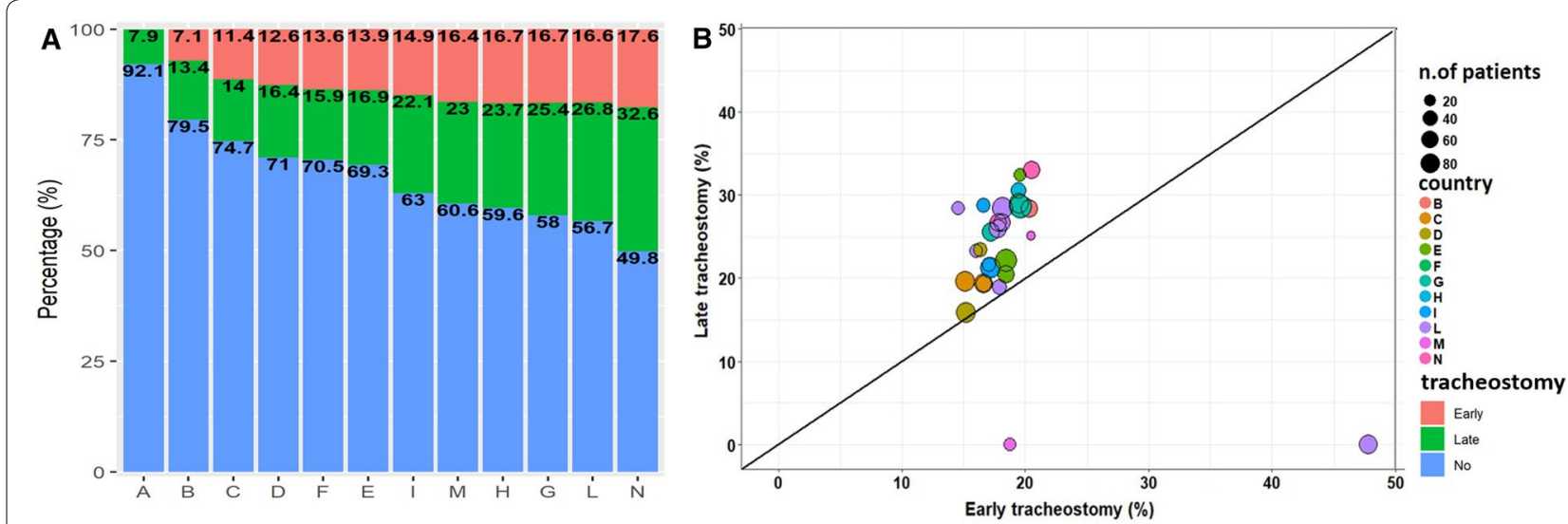

Fig. 1 Left panel (a). Percentage distribution of the decision to perform tracheostomy or not in each country (in blind). Only countries that have at least 20 patients admitted in ICU are reported alone; the remaining are grouped. Right panel (b). Percentage of early vs. late tracheostomy by centre with at least five tracheostomies. Centres within the same country have the same colour and a mass proportional to centre size. The bisector line is also reported. Results are adjusted for confounding factors 
Table 4 Results of the multivariable models on outcomes at 6 months or in ICU

\begin{tabular}{|c|c|c|c|c|c|c|c|c|c|c|c|c|}
\hline & \multicolumn{3}{|c|}{ GOSE $<5$ at 6 months } & \multicolumn{3}{|c|}{ Mortality at 6 months } & \multicolumn{3}{|c|}{ LOS in ICU } & \multicolumn{3}{|c|}{ LOS in hospital } \\
\hline & OR & $95 \% \mathrm{Cl}$ & $P$ value & HR & $95 \% \mathrm{Cl}$ & $P$ value & Coeff. & SE & $P$ value & Coeff. & SE & $\begin{array}{l}P \\
\text { value }\end{array}$ \\
\hline \multicolumn{13}{|l|}{ Model 1} \\
\hline Intercept & 0.13 & $0.05-0.31$ & $<0.001$ & & & & 21.38 & 2.79 & $<0.001$ & 40.96 & 5.93 & $<0.001$ \\
\hline Age (years) & 1.04 & $1.03-1.05$ & $<0.001$ & 1.06 & $1.04-1.07$ & $<0.001$ & -0.04 & 0.04 & 0.345 & -0.09 & 0.09 & 0.287 \\
\hline $\mathrm{GCS} \leq 8$ vs. $\mathrm{GCS}>8$ & 1.96 & $1.16-3.28$ & 0.006 & 1.35 & $0.78-2.34$ & 0.280 & 0.14 & 1.73 & 0.937 & -0.51 & 3.67 & 0.889 \\
\hline Pupils: $1-2$ unreactive vs. 2 reactive & 2.15 & $1.23-3.76$ & 0.004 & 2.30 & $1.38-3.80$ & 0.001 & 0.13 & 1.78 & 0.942 & 4.10 & 3.78 & 0.279 \\
\hline Late vs. early tracheostomy & 1.69 & $1.07-2.67$ & 0.018 & 1.22 & $0.73-2.03$ & 0.442 & 6.89 & 1.58 & $<0.001$ & 11.45 & 3.35 & $<0.001$ \\
\hline \multicolumn{13}{|l|}{ Model 2} \\
\hline Intercept & 0.11 & $0.05-0.27$ & $<0.001$ & & & & 19.91 & 2.76 & $<0.001$ & 38.55 & 5.89 & $<0.001$ \\
\hline Age (years) & 1.04 & $1.03-1.06$ & $<0.001$ & 1.06 & $1.04-1.08$ & $<0.001$ & -0.03 & 0.04 & 0.399 & -0.09 & 0.09 & 0.324 \\
\hline $\mathrm{GCS} \geq 8$ vs. GCS $<8$ & 1.96 & $1.16-3.29$ & 0.0065 & 1.30 & $0.76-2.24$ & 0.339 & 0.06 & 1.71 & 0.974 & -0.65 & 3.64 & 0.859 \\
\hline Pupils: $1-2$ unreactive vs. 2 reactive & 2.12 & $1.21-3.71$ & 0.0042 & 2.44 & $1.47-4.06$ & $<0.001$ & 0.02 & 1.75 & 0.992 & 3.91 & 3.74 & 0.296 \\
\hline Days waiting for tracheostomy & 1.04 & $1.01-1.07$ & 0.006 & 1.06 & $1.03-1.08$ & $<0.001$ & 0.52 & 0.09 & $<0.001$ & 0.86 & 0.19 & $<0.001$ \\
\hline
\end{tabular}

The influence of the timing of tracheostomy was evaluated with a categorical variable (Model 1) and as a discrete variable (Model 2)

Cl confidence interval, Coeff. coefficient, HR hazard ratio, LOS length of stay, OR odds ratio, SE standard error

tracheostomy was associated with an OR of 1.04 for unfavourable outcome (95\% CI=1.01-1.07, $p=0.006$ ). The multivariable Cox analysis on mortality at 6 months found that tracheostomy performed after 1 week was not associated with a significant increase of the hazard of mortality $(\mathrm{HR}=1.22,95 \% \mathrm{CI}=0.73-2.03 ; p=0.442)$. However, Model 2 showed that each increase of a day in the timing of tracheostomy was associated with a $6 \%$ increase in the hazard of mortality ( $\mathrm{HR}=1.06,95 \%$ $\mathrm{CI}=1.03-1.08, p<0.001)$. Late tracheostomy in Model 1 was associated with an increase in the mean ICU LOS of 6.9 days (95\% $\mathrm{CI}=3.7-9.9, p<0.001)$, and an increase in hospital LOS of 11.45 days $(95 \% \mathrm{CI}=4.88-18.02$, $p<0.001$ ); each 2 days deferral in tracheostomy was associated with a 1-day increase in ICU LOS, and a 2 day increase in hospital LOS. LOS after tracheostomy in ICU was shorter in the late tracheostomy group $(-2.33$ days, $p=0.04$ ), while the hospital LOS was similar between the two groups (ESM Table S5). Similar results were obtained when excluding ICU deaths (data not shown). Sensitivity analyses on all the outcomes considering complete data gave consistent results (ESM Table S4).

\section{Discussion}

At our knowledge, this analysis based on prospective observational data from CENTER-TBI [8] is the most extensive assessment of the practice of tracheostomy in TBI patients, across centres and countries in Europe. Our main findings are:
- Tracheostomy is commonly performed in TBI patients in ICU, and is most frequently undertaken after the first week in ICU;

- The likelihood of receiving a tracheostomy increases significantly with age, the severity of neurological injury (expressed as lower GCS and pupillary abnormalities), extra-cranial injury (particularly thoracic trauma), and early secondary insults (such as hypoxemia);

- There are significant variations in tracheostomy rates across countries and centres in Europe;

- When assessed as a discrete variable, later tracheostomies are associated with an increase in unfavourable outcome and LOS.

We found that tracheostomy was frequent amongst TBI patients in the ICU. The procedure was undertaken in $31.8 \%$ of our study cohort, which is more frequent than in studies in general ICU cohorts, where past literature reports rates of about $10 \%[16,17]$. This increased need for tracheostomy in the TBI population is attributable to a higher rate of extubation failure and the need for prolonged protection of the airways secondary to neurological injury. In general ICU patients, tracheostomy is most commonly performed after 14 days from admission [17, 18 , with only a quarter of tracheostomies delivered on or before day 7 [16]. In contrast, only $26 \%$ of our TBI cohort underwent tracheostomy later than 14 days from admission, and in $41 \%$, tracheostomy was undertaken before day 7.

The risk of receiving a tracheostomy was related to the severity of the neurological injury, quantified using GCS 
and pupillary reactivity at admission, and the presence of early secondary insults (such as hypoxemia). Non-neurological drivers of the decision to perform a tracheostomy include age and the occurrence of thoracic trauma, which may adversely affect respiratory weaning and extubation success. While the effect of non-neurological factors and hypoxemia on the risk of receiving tracheostomy was constant over time, the Cox model indicated that both GCS and pupillary reactivity had a time-dependent effect, with an increased impact on the HR of tracheostomy with increasing time from admission. These findings suggest that both the initial severity of the neurological injury and probably its trajectory, play a role in the decision process. The result that the median time to tracheostomy was 9 days post-admission probably reflects a change in treatment targets. In the initial phase, the aim is to manage acute intracranial emergencies, and tracheostomy at this stage could increase intracranial pressure and adversely affect the outcome. Once this phase is complete, cessation of sedation, weaning from ventilator support, and initiation of rehabilitation become key treatment targets. This timing of tracheostomy also prevents the use of the procedure in patients with lesser severities of injury, who might achieve successful extubation, and in those who have a rapidly progressive course and succumb early to their injuries. This process of selection still leads to tracheostomy at an earlier stage than commonly observed in non-TBI patients but allows the selection of a cohort most likely susceptible to the potential benefits of the procedure on the patients' outcomes $[19,20]$, by dealing with ongoing failure to protect the airway and the consequent risk of extubation failure [21-24].

However, the approach to tracheostomy was by no means uniform across ICUs that contributed to CENTER-TBI. We found substantial between-country and between-centre differences in the incidence and timing of tracheostomy, which persisted even after adjustment for covariates. Our results suggest that the current, local medical practices influence the decision to perform a tracheostomy, along with the ethical and legal implications context, clinical expertise, and costs relating to the procedure and equipment, replicating past findings in the general ICU population $[16,17,25]$.

The literature suggests that early tracheostomy may potentially reduce hospital stay, duration of mechanical ventilation and mortality rates [7, 19, 26, 27]. In a propensity-matched cohort study on TBI patients, early tracheostomy ( $\leq 7$ days) was associated with shorter mechanical ventilation duration (10 vs. 16 days, $\mathrm{RR}=0.70,95 \% \mathrm{CI}=0.66-0.75), \mathrm{ICU}$ and hospital LOS $(\mathrm{RR}=0.75, \quad \mathrm{CI}=0.66-0.75$, and $\mathrm{RR}=0.80,95 \%$ $\mathrm{CI}=0.74-0.86$ ), but did not affect mortality [28]. While the results of a Cochrane meta-analysis in general ICU patients [5] showed a possible mortality benefit from a tracheostomy, our data replicate smaller studies that specifically addressed TBI. Khalili et al. [20] found that, in a cohort of 152 TBI patients, early tracheostomy resulted in lower ICU and hospital LOS (46.6 vs. 38.6 days, $p=0.048$; and 34.9 vs. 26.7 days, $p=0.003$, respectively), but did not affect mortality. A meta-analysis by McCredie et al. [7] concluded that early tracheostomy might reduce the long-term mortality, duration of mechanical ventilation, and LOS. However, waiting longer, i.e., excluding patients probably improving or dying for brain damage, leads to fewer tracheostomy and similar short-term outcomes.

Each increase of 1 day in tracheostomy timing was significantly associated with a $4 \%$ increase in the risk of an unfavourable outcome with a $6 \%$ increase in the hazard of death. While this association may suggest a benefit from an earlier tracheostomy, we should be cautious about assigning causality to this association, since there may be competing confounds. Patients with more severe injury may have had a more prolonged need for therapies directed toward limiting the intracranial damage evolution (thus delaying tracheostomy) or might have a worse expected outcome (leading to a higher number of attempts to withhold tracheostomy).

In our cohort, patients who received late tracheostomy had a statistically significant longer mean LOS in ICU (by nearly 1 week) and in hospital (by about 11 days), with each 2 days deferral in tracheostomy associated with about 1 and 2 days' increase in LOS in ICU and hospital, respectively. In this direction also goes the interval between tracheostomy and discharge from ICU, which is shorter in the "later tracheostomy" group, along with the information that withdrawal of treatment is more frequent in patients without tracheostomy. Mortality in the ICU of tracheotomised patients was minimal (ESM figure S3).

\section{Limitations}

Although we used robust statistical methods and covariate adjustment, unidentified residual confounders may have affected our analyses. Moreover, although CENTERTBI banked detailed data on many aspects of injury, clinical care, and outcome, some key characteristics, such as those related to mechanical ventilation and respiratory complications, were not recorded. The observational nature of our study only allows us to report associations and cannot test the causal relationships between factors and tracheostomy practice.

\section{Conclusions}

Patients with TBI undergo a tracheostomy, more often than in general ICU populations. Several patient- and 
injury-related factors are associated with the decision to perform a tracheostomy in this group of patients. However, an analysis that adjusts for these covariates still shows substantial between-centre differences, which probably reflect inadequate evidence, a lack of consensus, and the absence of strong guidelines in this setting. The later performance of tracheostomy is associated with increased LOS and worse functional neurological outcome, but the causality of this relationship remains unproven. Randomized controlled trials exploring the effect of tracheostomy and its timing on patients' outcomes are warranted.

\section{Electronic supplementary material}

The online version of this article (https://doi.org/10.1007/s00134-020-05935-5) contains supplementary material, which is available to authorized users.

\section{Abbreviations}

ASAPS: American Society of Anaesthesiologists' physical status score; CENTERTBI: Collaborative European NeuroTrauma Effectiveness Research in Traumatic Brain Injury; Cl: Confidence interval; CT: Computed tomography; ESM: Electronic supplementary material; GCS: Glasgow coma scale; GOSE: Extended Glasgow Outcome Score; HR: Hazard ratio; HRS: Hours; ICP: Intracranial pressure; ICU: Intensive care unit; IQR: Interquartile range; ISS: Injury severity score; LOS: Length of stay; MOR: Median odds ratio; OR: Odds ratio; $\mathrm{PaO}_{2}$ : Partial pressure of oxygen; RR: Relative risk; $\mathrm{SaO}_{2}$ : Oxygen saturation; SD: Standard deviation; TBI: Traumatic Brain Injury; TV: Tidal volume; VAP: Ventilator-associated pneumonia.

\begin{abstract}
Author details
${ }^{1}$ Department of Anaesthesia and Intensive Care, Policlinico San Martino IRCCS for Oncology and Neuroscience, Genoa, Italy. ${ }^{2}$ School of Medicine and Surgery, University of Milano - Bicocca, Monza, Italy. ${ }^{3}$ Centre of Biostatistics for Clinical Epidemiology, University of Milano - Bicocca, Monza, Italy. ${ }^{4}$ Department of Public Health, Erasmus University Medical Center, Rotterdam, The Netherlands. ${ }^{5}$ Department of Physiopathology and Transplantation, Milan University, Milan, Italy. ${ }^{6}$ Neuro ICU Fondazione IRCCS Ca' Granda Ospedale Maggiore Policlinico, Milan, Italy. ${ }^{7}$ Department of Anaesthesia, University of Cambridge, Cambridge, UK. ${ }^{8}$ Neurointensive Care Unit, San Gerardo Hospital, ASST-Monza, Monza, Italy.
\end{abstract}

\section{Acknowledgements}

The CENTER-TBI ICU participants and investigators: Cecilia Åkerlund ${ }^{1}$ Krisztina Amrein ${ }^{2}$, Nada Andelic ${ }^{3}$, Lasse Andreassen ${ }^{4}$, Audny Anke ${ }^{5}$, Gérard Audibert ${ }^{6}$, Philippe Azouvi ${ }^{7}$, Maria Luisa Azzolini ${ }^{8}$, Ronald Bartels ${ }^{9}$, Ronny Beer $^{10}$, Bo-Michael Bellander ${ }^{11}$, Habib Benali ${ }^{12}$, Maurizio Berardino ${ }^{13}$, Luigi Beretta $^{8}$, Erta Biqiri ${ }^{14}$, Morten Blaabjerg ${ }^{15}$, Stine Borgen Lund ${ }^{16}$, Camilla Brorsson ${ }^{17}$, Andras Buki ${ }^{18}$, Manuel Cabeleira ${ }^{19}$, Alessio Caccioppola ${ }^{20}$, Emiliana Calappi $^{20}$, Maria Rosa Calvi ${ }^{8}$, Peter Cameron ${ }^{21}$, Guillermo Carbayo Lozano ${ }^{22}$, Marco Carbonara ${ }^{20}$, Ana M. Castaño-León ${ }^{23}$, Giorgio Chevallard ${ }^{14}$, Arturo Chieregato $^{14}$, Giuseppe Citerio ${ }^{24,25}$, Mark Coburn ${ }^{26}$, Jonathan Coles ${ }^{27}$, Jamie D. Cooper $^{28}$, Marta Correia ${ }^{29}$, Endre Czeiter ${ }^{18}$, Marek Czosnyka ${ }^{19}$, Claire Dahyot-Fizelier ${ }^{30}$, Véronique De Keyser ${ }^{31}$, Vincent Degos ${ }^{12}$, Francesco Della Corte $^{32}$, Hugo den Boogert ${ }^{9}$, Bart Depreitere ${ }^{33}$, Dula Dilvesi ${ }^{34}$, Abhishek Dixit $^{35}$ Jens Dreier ${ }^{36}$, Guy-Loup Dulière ${ }^{37}$, Ari Ercole ${ }^{35}$, Erzsébet Ezer $^{38}$, Martin Fabricius ${ }^{39}$, Kelly Foks ${ }^{40}$, Shirin Frisvold ${ }^{41}$, Alex Furmanov ${ }^{42}$, Damien Galanaud ${ }^{12}$, Dashiell Gantner $^{21}$, Alexandre Ghuysen ${ }^{43}$, Lelde Giga ${ }^{44}$, Jagos Golubovic ${ }^{34}$, Pedro A. Gomez ${ }^{23}$, Francesca Grossi ${ }^{32}$, Deepak Gupta ${ }^{45}$, lain Haitsma ${ }^{46}$, Raimund Helbok ${ }^{10}$, Eirik Helseth ${ }^{47}$, Peter J. Hutchinson ${ }^{48}$, Stefan Jankowski ${ }^{49}$, Mladen Karan ${ }^{34}$, Angelos G. Kolias ${ }^{48}$, Daniel Kondziella ${ }^{39}$, Evgenios Koraropoulos ${ }^{35}$, Lars-Owe Koskinen ${ }^{50}$, Noémi Kovács ${ }^{51}$, Ana Kowark ${ }^{26}$, Alfonso Lagares $^{23}$, Steven Laureys ${ }^{52}$, Aurelie Lejeune ${ }^{53}$, Roger Lightfoot ${ }^{54}$, Hester Lingsma ${ }^{55}$, Andrew I.R. Maas ${ }^{31}$, Alex Manara ${ }^{56}$, Costanza Martino ${ }^{57}$, Hugues Maréchal ${ }^{37}$, Julia Mattern ${ }^{58}$, Catherine McMahon ${ }^{59}$, David Menon ${ }^{35}$, Tomas Menovsky $^{31}$, Davide Mulazzi ${ }^{20}$, Visakh Muraleedharan ${ }^{60}$, Lynnette Murray ${ }^{21}$,
Nandesh Nair ${ }^{31}$, Ancuta Negru ${ }^{61}$, David Nelson ${ }^{1}$, Virginia Newcombe ${ }^{35}$, Quentin Noirhomme ${ }^{52}$, József Nyirádi ${ }^{2}$, Fabrizio Ortolano ${ }^{20}$, Jean-François Payen $^{62}$, Vincent Perlbarg ${ }^{12}$, Paolo Persona ${ }^{63}$, Wilco Peul ${ }^{64}$, Anna Piippo-Karjalainen ${ }^{65}$, Horia Ples ${ }^{61}$, Inigo Pomposo ${ }^{22}$, Jussi P. Posti ${ }^{66}$, Louis Puybasset ${ }^{67}$, Andreea Radoi ${ }^{68}$, Arminas Ragauskas ${ }^{69}$, Rahul Raj ${ }^{65}$, Jonathan Rhodes ${ }^{70}$, Sophie Richter $^{35}$, Saulius Rocka ${ }^{69}$, Cecilie Roe ${ }^{71}$, Olav Roise ${ }^{72,73}$, Jeffrey V. Rosenfeld ${ }^{74}$, Christina Rosenlund ${ }^{75}$, Guy Rosenthal ${ }^{42}$, Rolf Rossaint ${ }^{26}$, Sandra Rossi ${ }^{63}$, Juan ' Sahuquillo ${ }^{68}$, Oddrun Sandro ${ }^{76}$, Oliver Sakowitz ${ }^{58,77}$, Renan Sanchez-Porras ${ }^{77}$, Kari Schirmer-Mikalsen ${ }^{76,78}$, Rico Frederik Schou ${ }^{79}$, Peter Smielewski ${ }^{19}$, Abayómi Sorinola ${ }^{80}$, Emmanuel Stamatakis ${ }^{35}$, Ewout W. Steyerberg ${ }^{54,81}$, Nino Stocchetti ${ }^{82}$, Nina Sundström ${ }^{83}$, Riikka Takala ${ }^{84}$, Viktória Tamás ${ }^{80}$, Tomas Tamosuitis ${ }^{85}$, Olli Tenovuo ${ }^{66}$, Matt Thomas ${ }^{56}$, Dick Tibboe ${ }^{186}$, Christos Tolias ${ }^{87}$, Tony Trapani ${ }^{21}$, Cristina Maria Tudora ${ }^{61}$, Peter Vajkoczy ${ }^{88}$, Shirley Vallance ${ }^{21}$, Egils Valeinis ${ }^{44}$, Zoltán Vámos ${ }^{38}$, Gregory Van der Steen ${ }^{31}$, Jeroen T.J.M. van Dijck ${ }^{64}$, Thomas A. van Essen ${ }^{64}$, Audrey Vanhaudenhuyse ${ }^{12,52}$, Roel P. J. van Wijk $^{64}$, Alessia Vargiolu ${ }^{25}$, Emmanuel Vega ${ }^{53}$, Anne Vik ${ }^{78,89}$, Rimantas Vilcinis ${ }^{85}$, Victor Volovici ${ }^{46}$, Daphne Voormolen ${ }^{55}$, Petar Vulekovic ${ }^{34}$, Guy Williams ${ }^{35}$, Stefan Winzeck ${ }^{35}$, Stefan Wolf ${ }^{90}$, Alexander Younsi $^{58}$, Frederick A. Zeiler ${ }^{35,91}$, Agate Ziverte ${ }^{44}$, Tommaso Zoerle ${ }^{20}{ }^{1}$ Department of Physiology and Pharmacology, Section of Perioperative Medicine and Intensive Care, Karolinska Institutet, Stockholm, Sweden. ${ }^{2}$ János Szentágothai Research Centre, University of Pécs, Pécs, Hungary. ${ }^{3}$ Division of Surgery and Clinical Neuroscience, Department of Physical Medicine and Rehabilitation, Oslo University Hospital and University of Oslo, Oslo, Norway. ${ }^{4}$ Department of Neurosurgery, University Hospital Northern Norway, Tromso, Norway. ${ }^{5}$ Department of Physical Medicine and Rehabilitation, University Hospital Northern Norway, Tromso, Norway. ${ }^{6}$ Department of Anesthesiology \& Intensive Care, University Hospital Nancy, Nancy, France. ${ }^{7}$ Raymond Poincare hospital, Assistance Publique - Hopitaux de Paris, Paris, France. ${ }^{8}$ Department of Anesthesiology \& Intensive Care, S Raffaele University Hospital, Milan, Italy. ${ }^{9}$ Department of Neurosurgery, Radboud University Medical Center, Nijmegen, The Netherlands. ${ }^{10}$ Department of Neurology, Neurological Intensive Care Unit, Medical University of Innsbruck, Innsbruck, Austria. ${ }^{11}$ Department of Neurosurgery \& Anesthesia \& intensive care medicine, Karolinska University Hospital, Stockholm, Sweden. ${ }^{12}$ Anesthesie-Réanimation, Assistance Publique - Hopitaux de Paris, Paris, France. ${ }^{13}$ Department of Anesthesia \& ICU, AOU Città della Salute e della Scienza di Torino - Orthopedic and Trauma Center, Torino, Italy. ${ }^{14}$ Neurolntensive Care, Niguarda Hospital, Milan, Italy. ${ }^{15}$ Department of Neurology, Odense University Hospital, Odense, Denmark. ${ }^{16}$ Department of Public Health and Nursing, Faculty of Medicine and health Sciences,

Norwegian University of Science and Technology, NTNU, Trondheim, Norway. ${ }^{17}$ Department of Surgery and Perioperative Science, Umeå University, Umeå, Sweden. ${ }^{18}$ Department of Neurosurgery, Medical School, University of Pécs, Hungary and Neurotrauma Research Group, János Szentágothai Research Centre, University of Pécs, Hungary. ${ }^{19}$ Brain Physics Lab, Division of Neurosurgery, Dept of Clinical Neurosciences, University of Cambridge, Addenbrooke's Hospital, Cambridge, UK. ${ }^{20}$ Neuro ICU, Fondazione IRCCS Cà Granda Ospedale Maggiore Policlinico, Milan, Italy. ${ }^{21}$ ANZIC Research Centre, Monash University, Department of Epidemiology and Preventive Medicine, Melbourne, Victoria, Australia. ${ }^{22}$ Department of Neurosurgery, Hospital of Cruces, Bilbao, Spain. ${ }^{23}$ Department of Neurosurgery, Hospital Universitario 12 de Octubre, Madrid, Spain. ${ }^{24}$ School of Medicine and Surgery, Università Milano - Bicocca, Milano, Italy. ${ }^{25}$ Neurolntensive Care, ASST di Monza, Monza, Italy. ${ }^{26}$ Department of Anaesthesiology, University Hospital of Aachen, Aachen, Germany. ${ }^{27}$ Department of Anesthesia \& Neurointensive Care, Cambridge University Hospital NHS Foundation Trust, Cambridge, UK. ${ }^{28}$ School of Public Health \& PM, Monash University and The Alfred Hospital, Melbourne, Victoria, Australia. ${ }^{29}$ Radiology/MRI department, MRC Cognition and Brain Sciences Unit, Cambridge, UK. ${ }^{30}$ Intensive Care Unit, CHU Poitiers, Potiers, France. ${ }^{31}$ Department of Neurosurgery, Antwerp University Hospital and University of Antwerp, Edegem, Belgium. ${ }^{32}$ Department of Anesthesia \& Intensive Care, Maggiore Della Carità Hospital, Novara, Italy. ${ }^{33}$ Department of Neurosurgery, University Hospitals Leuven, Leuven, Belgium. ${ }^{34}$ Department of Neurosurgery, Clinical centre of Vojvodina, Faculty of Medicine, University of Novi Sad, Novi Sad, Serbia. ${ }^{35}$ Division of Anaesthesia, University of Cambridge, Addenbrooke's Hospital, Cambridge, UK. ${ }^{36}$ Center for Stroke Research Berlin, Charité - Universitätsmedizin Berlin, corporate member of Freie Universität Berlin, Humboldt-Universität zu Berlin, and Berlin Institute of Health, Berlin, Germany. ${ }^{37}$ Intensive Care Unit, CHR Citadelle, Liège, Belgium. ${ }^{38}$ Department of Anaesthesiology and Intensive Therapy, University of Pécs, Pécs, Hungary.

${ }^{39}$ Departments of Neurology, Clinical Neurophysiology and 
Neuroanesthesiology, Region Hovedstaden Rigshospitalet, Copenhagen, Denmark. ${ }^{40}$ Department of Neurology, Erasmus MC, Rotterdam, the Netherlands. ${ }^{41}$ Department of Anesthesiology and Intensive care, University Hospital Northern Norway, Tromso, Norway. ${ }^{42}$ Department of Neurosurgery, Hadassah-hebrew University Medical center, Jerusalem, Israel. ${ }^{43}$ Emergency Department, CHU, Liège, Belgium. ${ }^{44}$ Neurosurgery clinic, Pauls Stradins Clinical University Hospital, Riga, Latvia. ${ }^{45}$ Department of Neurosurgery, Neurosciences Centre \& JPN Apex trauma centre, All India Institute of Medical Sciences, New Delhi-1 10029, India. ${ }^{46}$ Department of Neurosurgery, Erasmus MC, Rotterdam, the Netherlands. ${ }^{47}$ Department of Neurosurgery, Oslo University Hospital, Oslo, Norway. ${ }^{48}$ Division of Neurosurgery, Department of Clinical Neurosciences, Addenbrooke's Hospital \& University of Cambridge, Cambridge, UK. ${ }^{49}$ Neurointensive Care, Sheffield Teaching Hospitals NHS Foundation Trust, Sheffield, UK. ${ }^{50}$ Department of Clinical Neuroscience, Neurosurgery, Umeå University, Umeå, Sweden. ${ }^{51}$ Hungarian Brain Research Program - Grant No. KTIA_13_NAP-A-II/8, University of Pécs, Pécs, Hungary. ${ }^{52}$ Cyclotron Research Center, University of Liège, Liège, Belgium. ${ }^{53}$ Department of AnesthesiologyIntensive Care, Lille University Hospital, Lille, France. ${ }^{54}$ Department of Anesthesiology \& Intensive Care, University Hospitals Southampton NHS Trust, Southampton, UK. ${ }^{55}$ Department of Public Health, Erasmus Medical Center-University Medical Center, Rotterdam, The Netherlands. ${ }^{56}$ Intensive Care Unit, Southmead Hospital, Bristol, Bristol, UK. ${ }^{57}$ Department of Anesthesia \& Intensive Care,M. Bufalini Hospital, Cesena, Italy. ${ }^{58}$ Department of Neurosurgery, University Hospital Heidelberg, Heidelberg, Germany. ${ }^{59}$ Department of Neurosurgery, The Walton centre NHS Foundation Trust, Liverpool, UK. ${ }^{60}$ Karolinska Institutet, INCF International Neuroinformatics Coordinating Facility, Stockholm, Sweden. ${ }^{61}$ Department of Neurosurgery, Emergency County Hospital Timisoara, Timisoara, Romania. ${ }^{62}$ Department of Anesthesiology \& Intensive Care, University Hospital of Grenoble, Grenoble, France. ${ }^{63}$ Department of Anesthesia \& Intensive Care, Azienda Ospedaliera Università di Padova, Padova, Italy. ${ }^{64}$ Dept. of Neurosurgery, Leiden University Medical Center, Leiden, The Netherlands and Dept. of Neurosurgery, Medical Center Haaglanden, The Hague, The Netherlands. ${ }^{65}$ Department of Neurosurgery, Helsinki University Central Hospital. ${ }^{66}$ Division of Clinical Neurosciences, Department of Neurosurgery and Turku Brain Injury Centre, Turku University Hospital and University of Turku, Turku, Finland. ${ }^{67}$ Department of Anesthesiology and Critical Care, Pitié -Salpêtrière Teaching Hospital, Assistance Publique, Hôpitaux de Paris and University Pierre et Marie Curie, Paris, France.

${ }^{68}$ Neurotraumatology and Neurosurgery Research Unit (UNINN), Vall d'Hebron Research Institute, Barcelona, Spain. ${ }^{69}$ Department of Neurosurgery, Kaunas University of technology and Vilnius University, Vilnius, Lithuania. ${ }^{70}$ Department of Anaesthesia, Critical Care \& Pain Medicine NHS Lothian \& University of Edinburg, Edinburgh, UK. ${ }^{71}$ Department of Physical Medicine and Rehabilitation, Oslo University Hospital/University of Oslo, Oslo, Norway. ${ }^{72}$ Division of Orthopedics, Oslo University Hospital, Oslo, Norway. ${ }^{73}$ Institute of Clinical Medicine, Faculty of Medicine, University of Olso, Oslo, Norway. ${ }^{74}$ National Trauma Research Institute, The Alfred Hospital, Monash University, Melbourne, Victoria, Australia. ${ }^{75}$ Department of Neurosurgery, Odense University Hospital, Odense, Denmark. ${ }^{76}$ Department of Anasthesiology and Intensive Care Medicine, St.Olavs. Hospital, Trondheim University Hospital, Trondheim, Norway. ${ }^{77}$ Klinik für Neurochirurgie, Klinikum Ludwigsburg, Ludwigsburg, Germany. ${ }^{78}$ Department of Neuromedicine and Movement Science, Norwegian University of Science and Technology, NTNU, Trondheim, Norway. ${ }^{79}$ Department of Neuroanesthesia and Neurointensive Care, Odense University Hospital, Odense, Denmark. ${ }^{80}$ Department of Neurosurgery, University of Pécs, Pécs, Hungary. ${ }^{81}$ Dept. of Department of Biomedical Data Sciences, Leiden University Medical Center, Leiden, The Netherlands. ${ }^{82}$ Department of Pathophysiology and Transplantation, Milan University, and Neuroscience ICU, Fondazione IRCCS Cà Granda Ospedale Maggiore Policlinico, Milano, Italy. ${ }^{83}$ Department of Radiation Sciences, Biomedical Engineering, Umeå University, Umeå, Sweden. ${ }^{84}$ Perioperative Services, Intensive Care Medicine and Pain Management, Turku University Hospital and University of Turku, Turku, Finland. ${ }^{85}$ Department of Neurosurgery, Kaunas University of Health Sciences, Kaunas, Lithuania. ${ }^{86}$ Intensive Care and Department of Pediatric Surgery, Erasmus Medical Center, Sophia Children's Hospital, Rotterdam, The Netherlands. ${ }^{87}$ Department of Neurosurgery, Kings college London, London, UK. ${ }^{88}$ Neurologie, Neurochirurgie und Psychiatrie, Charité - Universitätsmedizin Berlin, Berlin, Germany. ${ }^{89}$ Department of Neurosurgery, St.Olavs. Hospital, Trondheim University Hospital, Trondheim, Norway. ${ }^{90}$ Department of Neurosurgery, Charité - Universitätsmedizin Berlin, corporate member of Freie Universität Berlin, Humboldt-Universität zu Berlin, and Berlin Institute of
Health, Berlin, Germany. ${ }^{91}$ Section of Neurosurgery, Department of Surgery, Rady Faculty of Health Sciences, University of Manitoba, Winnipeg, MB, Canada.

\section{Authors' contributions}

GC ideated and supervised the project, participated in the data analysis, drafted the manuscript, and the supplementary tables, discussed the findings with all the authors, collected the COls. CR ideated the project, participated in the data analysis, drafted the manuscript, and the supplementary tables. FG and SG analysed the data, drafted the manuscript, and the supplementary tables. Cl was an active part of the manuscript drafting and revision. DM proofread the manuscript to ensure its compliance with standard scientific English'rules. All co-authors gave substantial feedback on the manuscript and approved the final version of it. This article is reported as per Strengthening the Reporting of Observational Studies in Epidemiology (STROBE) reporting guidelines (www.strobe-statemenent.org) (Electronic supplementary material ESM 1).

\section{Funding}

Data used in the preparation of this manuscript were obtained in the context of The Collaborative European NeuroTrauma Effectiveness Research in Traumatic Brain Injury (CENTER-TBI study, registered at clinicaltrials.gov NCT02210221), a large collaborative project, supported by the Framework 7 program of the European Union (602150). Additional funding was obtained from the Hannelore Kohl Stiftung (Germany), from OneMind (USA) and from Integra LifeSciences Corporation (USA). The funder had no role in the design of the study, the collection, analysis, and interpretation of data, or in writing the manuscript.

\section{Compliance with ethical standards}

\section{Conflicts of interest}

GC is Editor-in-Chief of Intensive Care Medicine. GC reports grants, personal fees as Speakers'Bureau Member and Advisory Board Member from Integra and Neuroptics; personal fees from Nestle and UCB Pharma, all outside of the submitted work. DKM reports grants from the European Union and UK National Institute for Health Research, during the conduct of the study; grants, personal fees, and non-financial support from GlaxoSmithKline; personal fees from Neurotrauma Sciences, Lantmaanen AB, Pressura, and Pfizer, outside of the submitted work. The other authors declare that they have no competing interests.

\section{Availability of data and materials}

The data supporting the findings in the study are available upon reasonable request from the corresponding Author (GC) and are stored at https://cente r-tbi.incf.org/_5cf8e3d1c3b0d43708ebef42. Imaging data can be found at https://center-tbi.incf.org/_5cf4dbd0560bb01102b6b28e, data on vitals values at https://center-tbi.incf.org/_5cf4dce9560bb01102b6b28f, while data regarding medications can be found at https://center-tbi.incf.org/_5cf4de0d56 obb01102b6b291.

\section{Ethics approval and consent to participate}

The Medical Ethics Committees of all participating centers approved the CENTER-TBI study, and informed consent was obtained according to local regulations.

\section{Publisher's Note}

Springer Nature remains neutral with regard to jurisdictional claims in published maps and institutional affiliations.

Received: 23 October 2019 Accepted: 11 January 2020

Published online: 5 February 2020

\section{References}

1. Rumbak MJ et al (2004) A prospective, randomized, study comparing early percutaneous dilational tracheotomy to prolonged translaryngeal intubation (delayed tracheotomy) in critically ill medical patients. Crit Care Med 32(8):1689-1694 
2. Raimondi $\mathrm{N}$ et al (2017) Evidence-based guidelines for the use of tracheostomy in critically ill patients. J Crit Care 38:304-318

3. Lazaridis C et al (2012) Liberation of neurosurgical patients from mechanical ventilation and tracheostomy in neurocritical care. J Crit Care 27(4):417 (e1-e8)

4. Siempos II et al (2015) Effect of early versus late or no tracheostomy on mortality and pneumonia of critically ill patients receiving mechanical ventilation: a systematic review and meta-analysis. Lancet Respir Med 3(2):150-158

5. Andriolo BNG et al (2015) Early versus late tracheostomy for critically ill patients. Cochrane Database Syst Rev 1(1):CD007271

6. Sewell MD et al (2018) Results of early and late surgical decompression and stabilization for acute traumatic cervical spinal cord injury in patients with concomitant chest injuries. World Neurosurg 118:e161-e165

7. McCredie VA et al (2017) Effect of early versus late tracheostomy or prolonged intubation in critically ill patients with acute brain injury: a systematic review and meta-analysis. Neurocrit Care 26(1):14-25

8. Steyerberg EW et al (2019) Case-mix, care pathways, and outcomes in patients with traumatic brain injury in CENTER-TBI: a European prospective, multicentre, longitudinal, cohort study. Lancet Neurol 18(10):923-934

9. Maas AIR et al (2014) Collaborative European NeuroTrauma effectiveness research in traumatic brain injury (CENTER-TBI): a prospective longitudinal observational study. Neurosurgery 76(1):67-80

10. Wilson JTL, LE Pettigrew, Teasdale GM (1998) Structured interviews for the glasgow outcome scale and the extended glasgow outcome scale: guidelines for their use. J Neurotrauma 15(8):573-585

11. Watkins JF, Valsecchi M-G (1996) Analysing survival data from clinical trials and observational studies. Statistics 45:391

12. Merlo J et al (2006) A brief conceptual tutorial of multilevel analysis in social epidemiology: using measures of clustering in multilevel logistic regression to investigate contextual phenomena. J Epidemiol Community Health 60(4):290-297

13. Marmarou A et al (2007) IMPACT database of traumatic brain injury: design and description. J Neurotrauma 24(2):239-250

14. Zhang Z (2016) Multiple imputation with multivariate imputation by chained equation (MICE) package. Ann TransI Med 4(2):30
15. Computing., R.F.f.S. (ed) (2019) R: A language and environment for statistical computing. R.F.f.S. Computing, Austria

16. Abe T et al (2018) Epidemiology and patterns of tracheostomy practice in patients with acute respiratory distress syndrome in ICUs across 50 countries. Crit Care 22(1):195

17. Mehta AB et al (2015) Trends in tracheostomy for mechanically ventilated patients in the United States, 1993-2012. Am J Respir Crit Care Med 192(4):446-454

18. Navalesi P et al (2008) Rate of reintubation in mechanically ventilated neurosurgical and neurologic patients: evaluation of a systematic approach to weaning and extubation. Crit Care Med 36(11):2986-2992

19. Frutos-Vivar F et al (2005) Outcome of mechanically ventilated patients who require a tracheostomy. Crit Care Med 33(2):290-298

20. Khalili $\mathrm{H}$ et al (2017) Experience with traumatic brain injury: Is early tracheostomy associated with better prognosis? World Neurosurg 103:88-93

21. Wang Y et al (2018) A meta-analysis of the influencing factors for tracheostomy after cervical spinal cord injury. Biomed Res Int 2018:5895830

22. Bosel $J$ et al (2013) Stroke-related early tracheostomy versus prolonged orotracheal intubation in Neurocritical Care Trial (SETPOINT): a randomized pilot trial. Stroke 44(1):21-28

23. Maclntyre N (2007) Discontinuing mechanical ventilatory support. Chest 132(3):1049-1056

24. Blot F et al (2008) Early tracheotomy versus prolonged endotracheal intubation in unselected severely ill ICU patients. Intensive Care Med 34(10):1779-1787

25. Durbin CG Jr (2005) Indications for and timing of tracheostomy. Respir Care 50(4):483-487

26. Bouderka MA et al (2004) Early tracheostomy versus prolonged endotracheal intubation in severe head injury. J Trauma 57(2):251-254

27. Sugerman $\mathrm{HJ}$ et al (1997) Multicenter, randomized, prospective trial of early tracheostomy. J Trauma 43(5):741-747

28. Alali AS et al (2014) Tracheostomy timing in traumatic brain injury: a propensity-matched cohort study. J Trauma Acute Care Surg 76(1):70-76 